\title{
Rotational Path Removable Partial Denture (RPD): Conservative Esthetic Treatment Option for the Edentulous Mandibular Anterior Region: A Case Report
}

JENNIFER S. SUH, DMD*

EDWARD J. BILLY, DMD ${ }^{\dagger}$

\begin{abstract}
It can be esthetically and financially daunting for patients to lose teeth in an anterior region of the mouth. For these patients, traditional treatment options presented in the past have included fixed partial denture, implants, and conventional removable partial denture (RPD). For patients faced with financial, anatomical, and/or esthetic limitations, the edentulous region can be restored successfully with a rotational path RPD. Rotational path RPD designs have often been overlooked by the dental profession due to its complex concepts involving the prosthetic design and sensitive laboratory techniques. With better understanding of the concepts and design, the dental clinician can deliver the highest esthetic outcome in compromised areas in which other treatment options may often face limitations. This paper reviews the method used to esthetically design and plan a posterior-anterior rotational path RPD in an edentulous mandibular anterior region for a patient missing the mandibular incisors.
\end{abstract}

\section{CLINICAL SIGNIFICANCE}

Due to inadequate understanding of the mechanics of rotational path RPDs, many clinicians have not adapted the application of this advantageous prosthesis. When correctly designed and fabricated, the rotational path RPD provides improved esthetics, cleanliness, and retention for patients who may not be suitable candidates for implants or fixed partial dentures in toothsupported edentulous regions.

(J Esthet Restor Dent 20:98-107, 2008)

INTRODUCTION

$\mathrm{D}$ espite the advanced techniques available to replace edentulous areas with implant therapy, there remains a group of patients who are not good candidates for implant therapy.
This group of patients may lack financial resources and may have poor systemic health, fear of dental surgeries, and psychological or anatomical limitations. ${ }^{1}$ Patients who fit into the aforementioned categories have the option of receiving a removable partial denture (RPD) prosthesis to replace missing teeth. In place of the sometimes cumbersome and unesthetic conventional RPD design for replacing teeth in the missing mandibular anterior region, the rotational path RPD has become a popular treatment option. The 
rotational path prosthesis offers the advantage of improved esthetics by eliminating anterior clasps, shortened treatment duration, cleanliness, and lower treatment cost over implants or fixed partial dentures. ${ }^{1-3}$ The need for an esthetic RPD involving anterior pontics gave rise to the dual path RPD concept first introduced by King and Garver in 1978. ${ }^{3}$ By eliminating anterior clasping, this technique utilizes proximal undercuts adjacent to the edentulous spaces for retention. ${ }^{2,4-6}$ In the beginning, the dual path design was limited to tooth-borne situations where anterior teeth were missing; however, clinicians are applying the concept to other edentulous spaces. ${ }^{4}$ This article reviews the method used to esthetically design and plan a posterioranterior (PA) rotational path RPD in an edentulous mandibular anterior region for a patient missing mandibular incisors.

BASIC ROTATIONAL PATH RPD CONCEPT

The rotational path design concept uses a rigid retainer portion of the framework as the retention component. ${ }^{2,5,6}$ The anterior-posterior, PA, and lateral rotational path designs are popularly used in Kennedy Class IV situations. ${ }^{1}$ The proximal plate provides retention through its intimate contact with the proximal tooth surface below the height of contour at a zero-degree tilt. ${ }^{2}$ These rigid retentive components gain access along the first path, to engage into the undercuts, and then are maneuvered into rotation to fully seat the prosthesis-the second and completed path of insertion. ${ }^{2,3}$ Only a minor connector, a guiding plane, or a small rigid extension of the framework into a desired undercut is required for retention. ${ }^{5}$ The effectiveness of the rotational path design is dependent on the one or two conventional clasp assemblies placed on the prosthesis. ${ }^{2}$

PARAMETERS OF ROTATIONAL PATH RPD

According to Krol and colleagues, ${ }^{6}$ there are more advantages than disadvantages in utilizing rotational path RPD. Clinicians should evaluate patients on an individual basis and determine if a rotational path design is possible.

The rotational path RPD design offers many advantages. ${ }^{6}$ It requires minimal number of clasps, and hence, reduces tooth coverage and decreases plaque accumulation. By eliminating anterior clasps, the esthetics can be better manipulated, compared with fixed partial dentures, around the gingival defects, with closer acrylic adaptation. The abutment teeth preparation is conservative compared with fixed prosthesis or a precision attachment, and the design does not require lingual or facial undercuts. Also, rotational path RPD prevents further tipping of abutment teeth contacted by the rigid retainer. Despite many of these advantages, the rotational path RPD design also has several disadvantages. ${ }^{6}$ Communication with a competent laboratory technician is crucial, as the rigid retentive component is difficult to adjust and the RPD is less tolerant to any fabrication and processing errors. Lastly, the design requires wellprepared rest seats.

CLINICAL APPLICATION: CASE STUDY

\section{History}

A 72-year-old White male presented to the graduate prosthodontics clinic of the University of Michigan dental school seeking an evaluation for ways to improve function and esthetics. The patient was given a fixed (FPD \#22-26 and implant FPD \#23-26) versus RPD treatment option and decided to accept the removable treatment option. The patient's goal was to receive a comfortable and functional prosthesis with no surgical treatment. His medical history revealed no significant medical findings and his general health was excellent. During the clinical examination, the following problems were noted: teeth \#5 and \#23 to \#26 were missing, and teeth \#18 and \#31 presented with existing gold crowns. The patient was not interested in replacing tooth \#5 at the present 


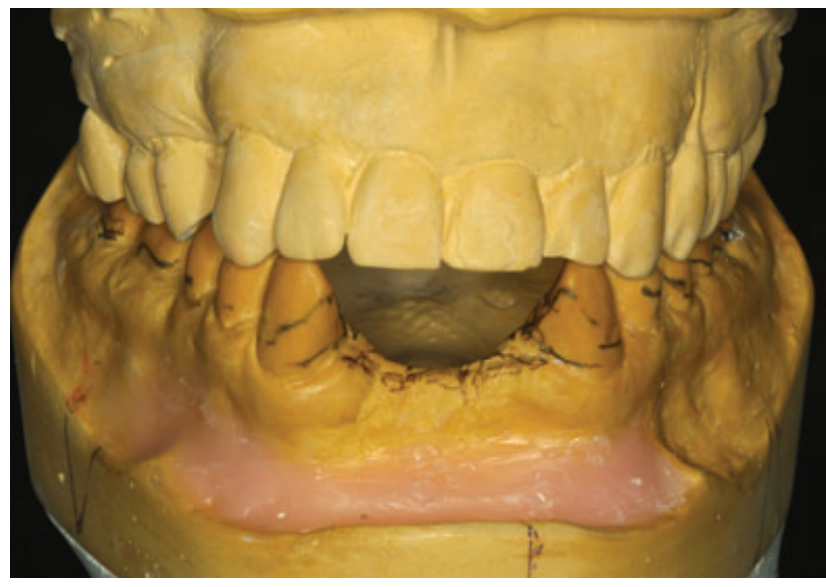

Figure 1. Importance of mounted casts for interocclusal space evaluation.

time. Rest seat preparations in the existing gold crowns were to be prepared with no perforation after careful evaluation of occlusal relationship in the mounted casts. The patient exhibited canine guidance occlusal relationship bilaterally and exhibited no parafunctional habits. His acceptance to the removable prosthesis treatment plan was very philosophical according to the House's classification, and the treatment was projected to have a good longterm prognosis.

\section{CLINICAL PROCEDURE}

\section{Diagnosis and Treatment Plan}

A facebow (Water Pik Tech Inc., Newport Beach, CA, USA) was used to help relate the diagnostic casts, which were mounted onto a semiadjustable articulator (Hanau Wide Vue-Articulator II and facebow, Water Pik, Inc., Fort Collins, CO, USA). It was indicated.

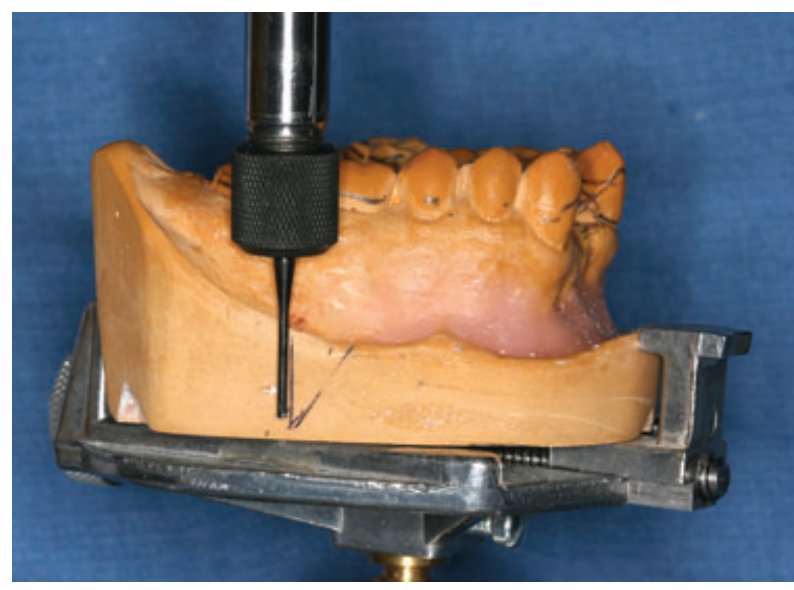

Figure 2. Tripodized casts at zero degrees.

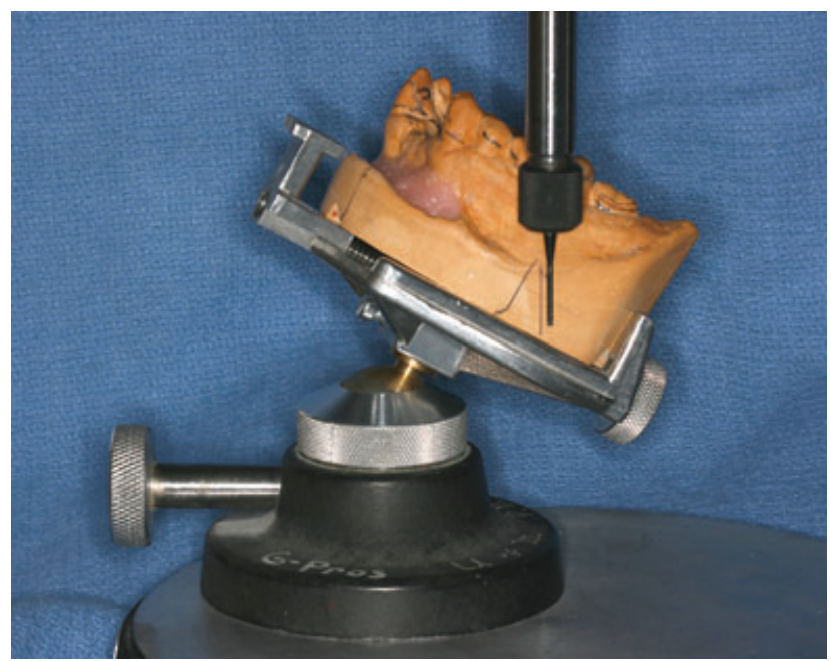

Figure 3. The direction of the first path of insertion

important to have mounted casts to evaluate the available interarch space and to determine the appropriate surfaces to place occlusal rest seats (Figure 1).

\section{Survey Casts}

The mandibular cast was placed on a surveyor table (Dentsply Ceramco, Ney Surveyor, Yucaipa,
CA, USA) and was surveyed at a zero-degree tilt. The analyzing rod was used to determine the amount of undercut present on the mesial surface of the canines and mesial-lingual undercut on molars. Three separate vertical lines were placed on the cast to orient it at a zero-degree tilt (Figures 2 and 3). The cast was 


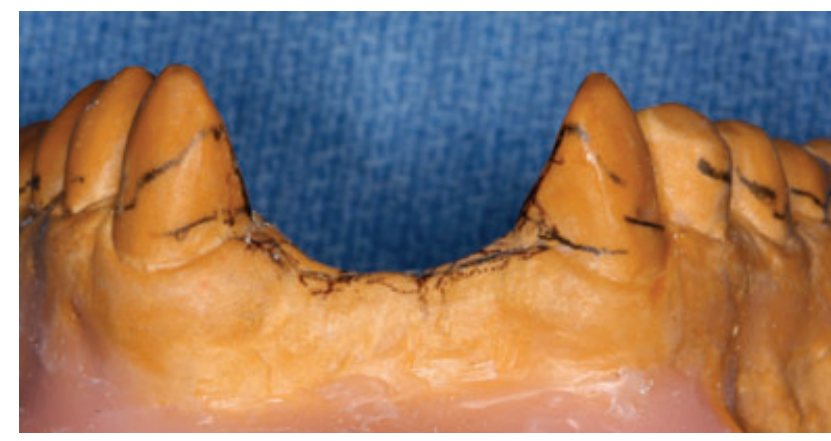

Figure 4. The distance between the two heights of contour on the canine should be as far apart as possible for better retention.

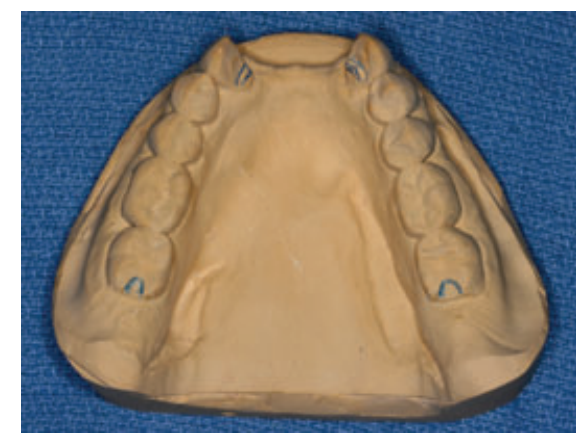

Figure 5. Composite cingulum rest seats were placed on canines. tilted straight back from the initial survey lines until the undercuts on the mesial surfaces of the canines were eliminated. Once the tilt on the surveyor was determined, three additional vertical lines were drawn next to the initial vertical lines (Figure 3). The RPDs will have better retention the more divergent the survey lines are from each other. The preliminary height of contour outline was drawn on the diagnostic cast, and the following modification areas were indicated on the cast: rest seats, retentive areas, undercuts, and guiding planes for completion with the patient (Figures 4 and 5).

\section{RPD Framework Design}

Major framework rest seats and tooth modifications were completed on the patient by building up Gradia composite (GC-America, Alsip, IL, USA) cingulum rest seats $^{7}$ on the lingual surfaces of canines, and disto-occlusal rest

\begin{tabular}{|c|c|c|c|}
\hline Teeth & Rest & Undercut & Clasps \\
\hline 22 & Cingulum & $\begin{array}{c}\text { No electroplating on mesial guide } \\
\text { plane; block out lingual rest }\end{array}$ & NA \\
\hline 27 & Cingulum & $\begin{array}{c}\text { No electroplating on mesial guide } \\
\text { plane; block out lingual rest }\end{array}$ & NA \\
\hline 18 & $\mathrm{DO}$ & ML & Aker's ( $1 / 2$ round) \\
\hline 31 & DO & ML & Aker's ( $1 / 2$ round) \\
\hline \multicolumn{4}{|c|}{$\begin{array}{l}\text { Major connector: lingual bar. } \\
\text { RPD metal framework: Wironium (Zedan Lab, Farmington Hills, MI, USA). } \\
\text { Retentive area: lattice design. }\end{array}$} \\
\hline
\end{tabular}

seats $(1.5 \text {-mm-deep })^{2}$ were prepared with an inverted-cone diamond bur on teeth \#18 and \#31 (Figure 5, Table 1). Although full-coverage survey crowns may be more beneficial to prevent composite wear against the mechanical friction of the prosthesis over time, many authors ${ }^{8-10}$ have advocated bonded restorations to improve contours and to alleviate the patient's financial constraints.

\section{Custom Tray Fabrication and} Definitive PVS Impression The flange portion of a Triad custom tray (Dentsply Trubyte, York, PA, USA) was bordermolded using green stick compound (Kerr Dental, Orange, CA, USA). The definitive cast impression was taken using a light-body Extrude polyvinylsiloxane (Kerr Dental) to capture the details of the rest seats and a heavy body to fill the rest of the tray

(Figure 6). 


\section{Prepared Master Cast Sent to the Laboratory}

After the master cast was poured, trimmed, and resurveyed, a laboratory authorization was written to communicate with the laboratory for the fabrication of the framework. It was important to specify not to provide relief around the mesial guide planes of the abutment teeth \#22 and \#27, as this was critical for allowing rigid retainers to engage in undercuts (Figure 7). It is important to instruct the laboratory not to electropolish the guide plane areas in order to prevent the loss of retention. For rotational path RPDs, each retainer consists of a rest and a guide plane as the retentive element, which avoids the use of clasps, especially in the esthetic zone.

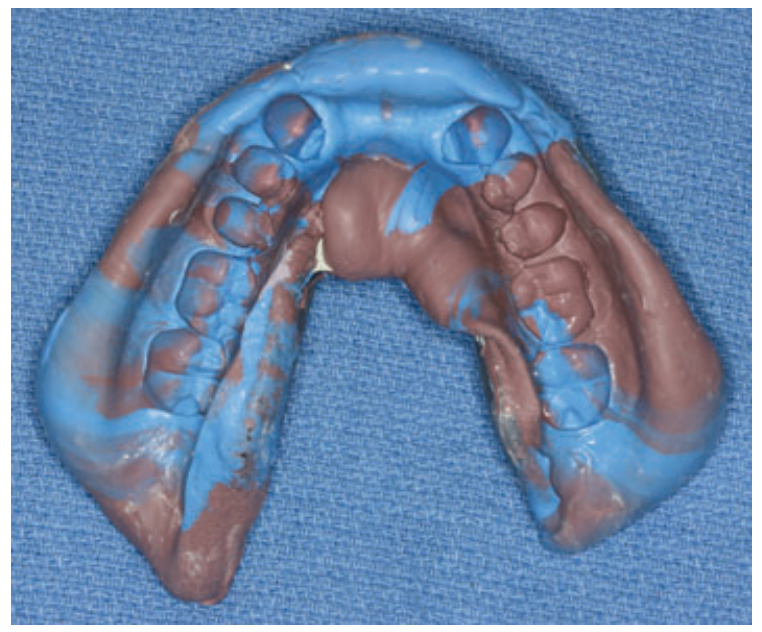

Figure 6. PVS impression with a custom tray.

\section{Framework Try-In}

The framework was tried in the mouth to check for retention by pulling on the meshwork area to see if it unseats and to evaluate the proper sitting of the RPD around the rest seats, guide plane, and clasps and the fit of major connectors (Figure 7).

\section{Set Selected Teeth according to Shade and Shape \\ Correct shade and shape of the teeth were selected by evaluating the patient's remaining dentition as a guide. Gingival shade was also taken at the same appointment.}

\section{Mount Mandibular Cast against the Maxillary Cast}

Mandibular cast was mounted to the opposing cast in order to wax-up the edentulous area.
A

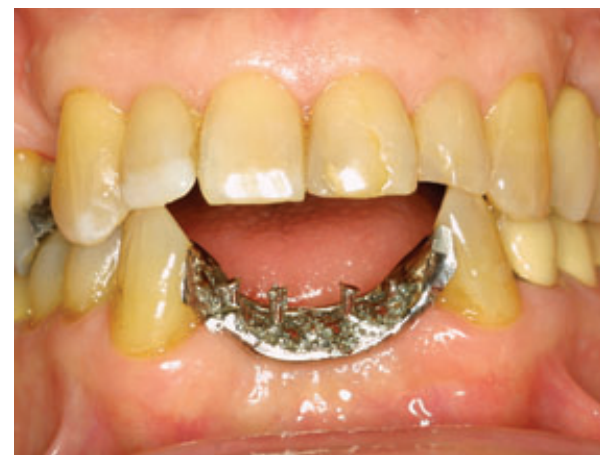

B

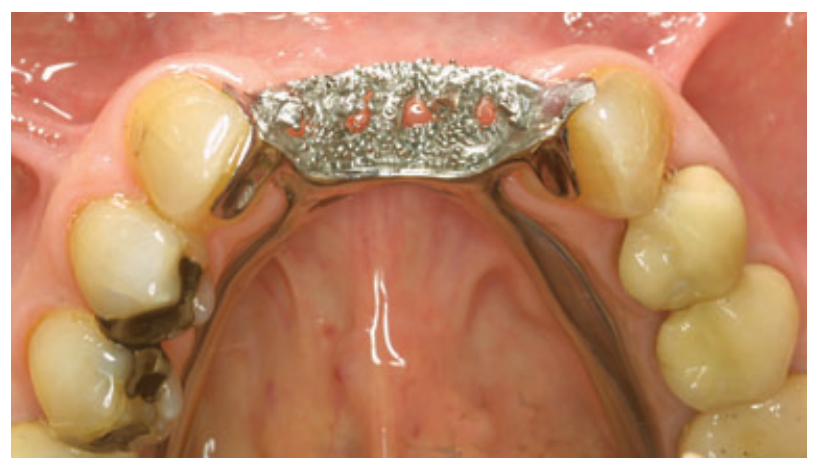

Figure 7. Intimate adaptation of framework around the abutment teeth. 
A

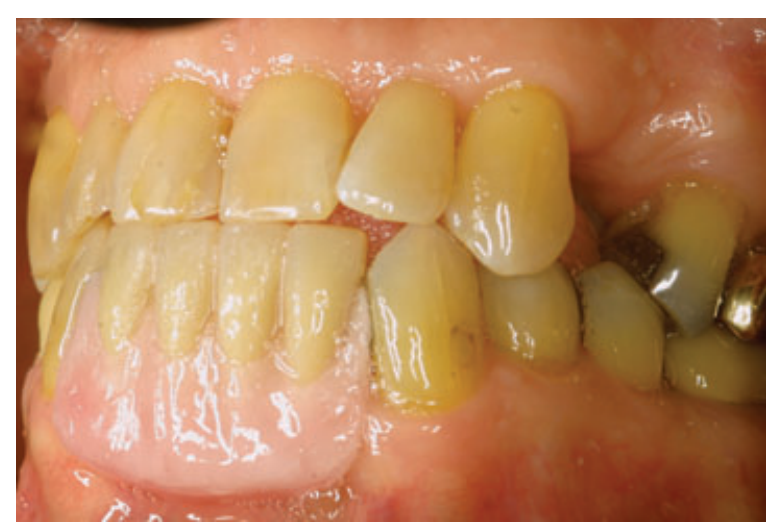

B

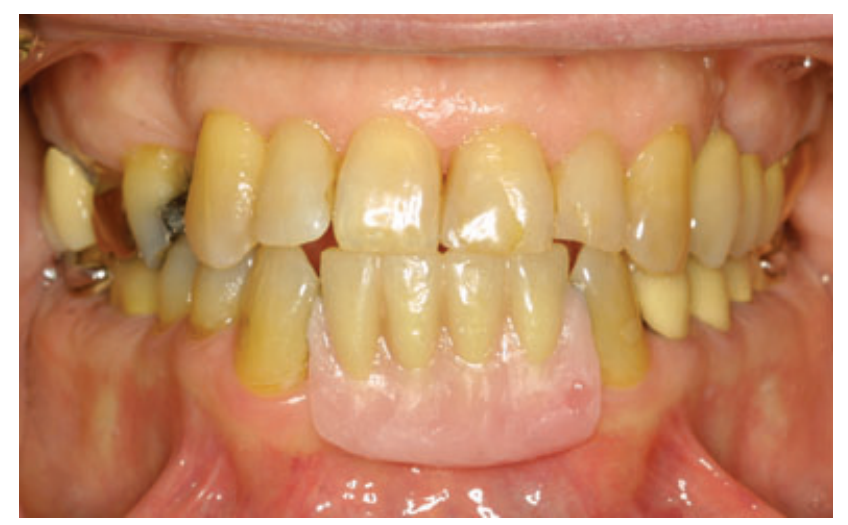

Figure 8. Wax try-in.

A

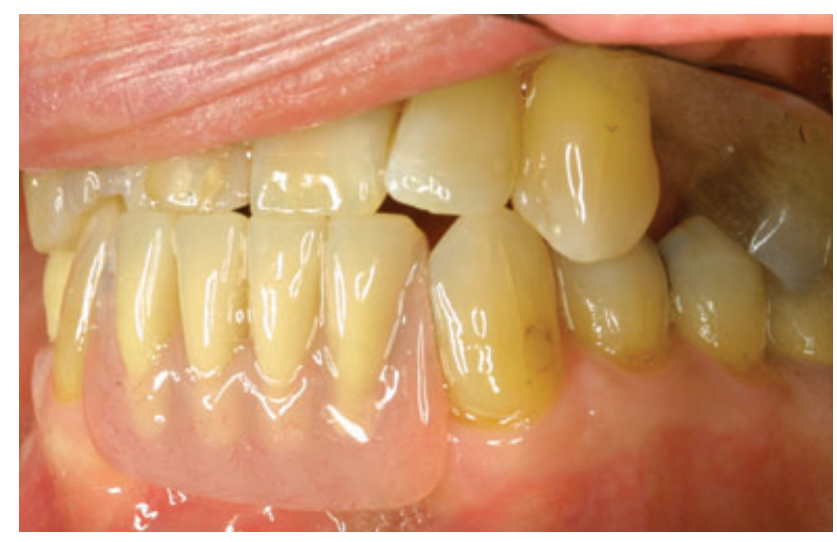

B

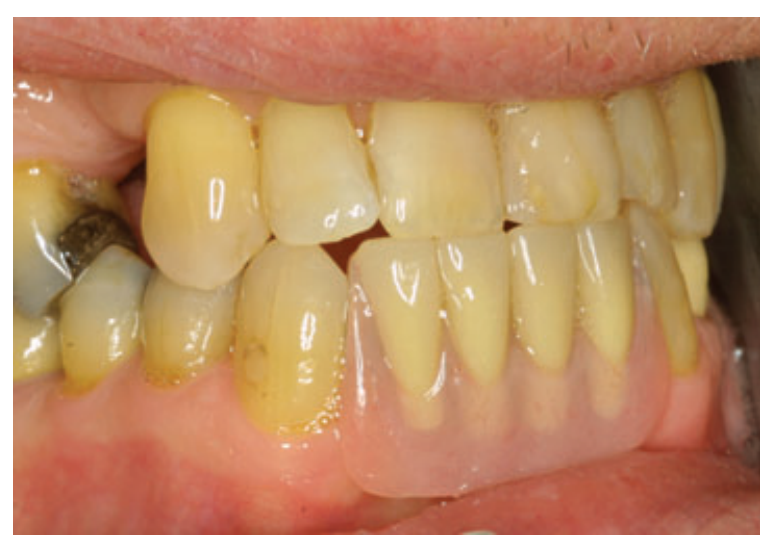

Figure 9. Delivery of final prosthesis. Notice the adaptation of acrylic resin to the adjacent tooth.

\section{Esthetic Try-In}

Teeth have been set and waxedup for the esthetic try-in appointment. The RPD was gently placed in the mouth, and the following criteria were evaluated: height of the teeth, occlusal interferences, esthetics, and phonetics (Figure 8).

\section{Wax-Up, Process RPD}

After obtaining the patient's approval for the wax-up and tooth setup, the RPD was processed using light-fibered pink Lucitone (Dentsply Trubyte).

\section{Placement of Definitive RPD}

The processed RPD was placed in the mouth, and the following criteria were reevaluated: adaptation of the clasps and rests, retention of the RPD, esthetics, and occlusion (Figures 9 and 10).

\section{Patient Education}

The patient was instructed in placing the prosthesis by seating the posterior part of the RPD first and then seating the anterior section until it clicked into the edentulous area by utilizing the PA rotational path. Oral hygiene instruction was provided, which stressed the importance of recall maintenance appointments to evaluate the prosthesis as 
A

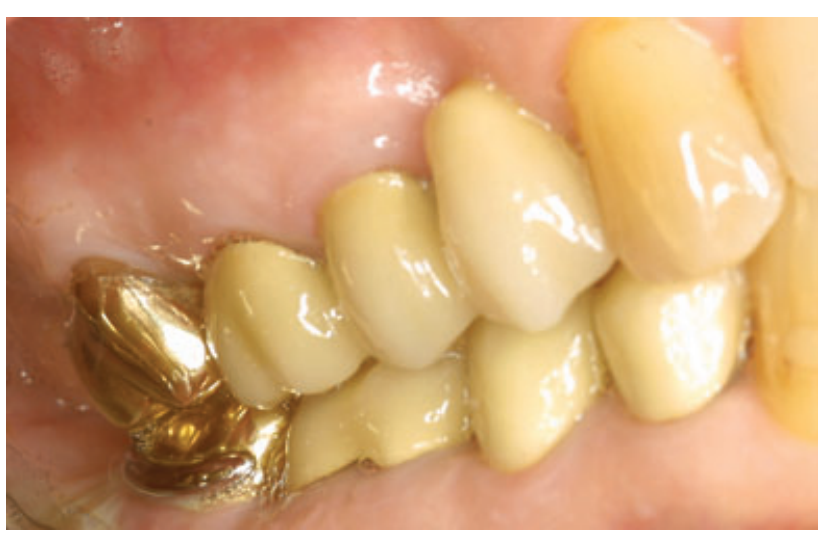

B

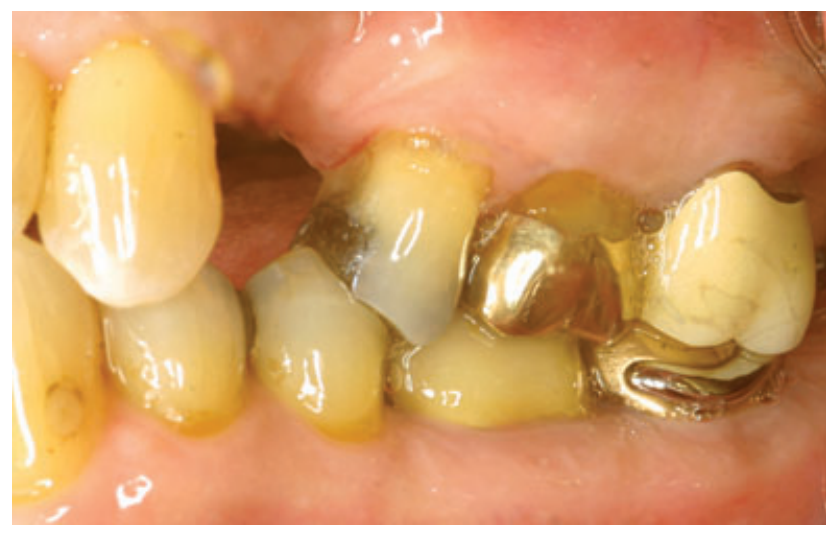

Figure 10. C-clasp adaptation around teeth \#18 and \#31.

well as the health of the remaining dentition.

\section{CONCLUSION}

With partially edentulous patients, clinicians are challenged to improve on treatment plans and utilize alternative RPD designs to provide comfort and to meet the current esthetic dental standards. The rotational path of insertion concept satisfies this current need of patients opting to receive a removable treatment plan. ${ }^{1}$ By rotating an RPD into position, fewer clasps are utilized. A careful examination of the patient's existing dentition can allow a rotational path RPD to be designed properly. Rotational path RPD can provide an alternative treatment option to implants for medically compromised patients who cannot go through the surgical phases of implant therapy. Rotational path $\mathrm{RPD}$ is clinically significant in that it can deliver satisfactory esthetics and function for patients that is comparable with fixed partial denture or implant therapy. ${ }^{1,6}$ By utilizing the existing undercuts present in the dentition, rotational path RPDs can be inserted using a dual path placement. One of the main advantages of rotational path RPDs is the claspless design in the esthetic region. Because of the close adaptation requirements of retentive components, an intimate tooth contact by the rests allows very little room for error while the RPD is fabricated. ${ }^{6}$ It is important for the clinician to build a close relationship and communicate well with the laboratory technician for the successful fabrication of a rotational path RPD. ${ }^{6}$ It seems that many dentists have a fear or lack of trust in their ability to deliver a predictable RPD prosthesis, and thus avoid recommending this treatment to their patients. ${ }^{5}$ The long-term success of an RPD can be very predictable with the proper attention to oral hygiene, periodontal considerations, basic design concepts, and judicious fabrication of partial denture construction. ${ }^{4}$

DISCLOSURE AND ACKNOWLEDGMENTS

The authors do not have any financial interest in the companies whose materials are included in this article.

The authors gratefully acknowledge the support and encouragement given by Dr. Michael Razzoog.

\section{REFERENCES}

1. Ancowitz S. Esthetic removable partial dentures. Gen Dent 2004;42(5):452-3.

2. Jacobson TE, Krol AJ. Rotational path removable partial denture 
design. J Prosthet Dent 1982;48(4):370-6.

3. King G. Dual-path design for removable partial dentures. J Prosthet Dent 1978;39(4):392-5.

4. Becker CM, Kaiser DA, Goldfogel MH. Evolution of removable partial denture design. J Prosthodont 1994;3(3): 158-66.

5. Christensen GJ. What has happened to removable partial prosthodontics? JADA 2003;134:111-3.

6. Krol AJ, Jacobson TE, Finzen FC. Removable partial denture design. 5th ed.
San Rafael (CA): Indent; 1999, pp. 78-93.

7. Toth RW, Fiebiger GE, Mackert JR Jr., Goldman BM. Shear strength of lingual rest seats prepared in bonded composite. J Prosthet Dent 1986;56(1):99-104.

8. Latta GH. A technique for preparation of lingual rest seats in light-cured composite. J Prosthet Dent $1988 ; 60(1) ; 127$.

9. Wong R, Nicolls JI, Smith DE. Evaluation of prefabricated lingual rest seats for removable partial dentures. J Prosthet Dent 1982;48(5):521-6.
10. Yard RA, Butler GV, Render PJ. Bonded composite rests: a fabrication method. J Prosthet Dent 1988;60(1):128-9.

Reprint requests: Jennifer S. Suh, DMD, Department of Biologic and Material Science, The University of Michigan School of Dentistry, Graduate Prosthodontics, Room 1378, 1011 N. University, Ann Arbor, MI 48109-1078. Tel.: 734-763-3326; Fax: 734-764-1481; e-mail: jsuhdmd@ umich.edu 